\title{
Targeted imaging of human endothelial-specific marker in a model of adoptive cell transfer
}

\author{
Hye Won Kang ${ }^{1}$, Denise Torres ${ }^{1}$, Lawrence Wald ${ }^{2}$, Ralph Weissleder ${ }^{1}$ and \\ Alexei A Bogdanov Jr${ }^{1}$ \\ ${ }^{1}$ Center for Molecular Imaging Research, Department of Radiology, Massachusetts General Hospital and \\ Harvard Medical School, Charlestown, MA, USA and ${ }^{2}$ Athinoula A. Martinos Center for Biomedical Imaging, \\ Department of Radiology, Massachusetts General Hospital, Charlestown, MA, USA
}

\begin{abstract}
Imaging of endothelial-specific markers is critically important in non-invasive detection of early signs of vascular pathologies (eg inflammation, atherosclerosis and angiogenesis). A model of adoptive human endothelial cell (HUVEC) transfer was used to test-specific imaging probes for human vascular disease consisting of cross-linked iron oxide (CLIO) nanoparticles conjugated to anti-human E-selectin (CLIO-F(ab') $)_{2}$ ). To perform in vivo imaging of E-selectin expression in functional blood vessels, human vascular endothelium cells (HUVECs) were implanted in athymic mice in Matrigel solution, which served as a temporary neovascularization scaffold after the solidification. The formation of HUVEC-containing vessels was established by histology and microscopy. CLIO-F(ab') $)_{2}$ probes were administered via an i.v. injection following the induction of E-selectin expression by IL-1 $\beta$. High-resolution MR images were obtained before and after the administration of CLIO-F(ab' $)_{2}$, which showed specific hypointensity only if treated with IL-1 $\beta$. A three-times higher CLIO-induced MR signal decrease on T2* images was measured in HUVEC implants in response to IL-1 $\beta$ treatment. Image signal intensity did not change in control animals that: (1) harbored Matrigel alone, (2) in the absence of IL-1 $\beta$ treatment or (3) in animals injected with CLIO linked to the idiotype-matched control $F\left(a b^{\prime}\right)_{2}$. Experiments in an adoptive transfer model demonstrated that HUVEC-containing neovessels are perfused and that IL-1 $\beta$ inducible E-selectin expression in these vessels is detectable with non-invasive imaging by using targeted nanoparticles.
\end{abstract}

Laboratory Investigation (2006) 86, 599-609. doi:10.1038/labinvest.3700421; published online 10 April 2006

Keywords: E-selectin; endothelium; adoptive transfer; superparamagnetic iron oxide nanoparticles; MRI

Non-invasive imaging of endothelial cell surface markers holds promise in identifying early signs of inflammation, angiogenesis or atherosclerosis in vivo. E-selectin (ELAM-1), a proinflammatory molecule involved in rolling of leukocytes on endothelium in inflammation, ${ }^{1}$ is upregulated in proliferating endothelial cells under non-inflammatory conditions. ${ }^{2}$ E-selectin upregulation by VEGF $^{3}$ and endogenous IL- $1 \alpha^{4}$ suggests a role in tumor angiogenesis and metastasis ${ }^{5}$ as the presence of soluble variant of E-selectin in human plasma correlated with severity of breast cancer. ${ }^{6}$ It has

Correspondence: Dr A Bogdanov Jr, PhD, Department of Radiology and Cell Biology, University of Massachusetts Medical School, S2-804, 55 Lake Avenue North, Worcester, MA 01655, USA.

E-mail: Alexei.Bogdanov@umassmed.edu

Received 1 December 2005; revised 11 March 2006; accepted 12 March 2006; published online 10 April 2006 been previously suggested that E-selectin induces angiogenesis $^{7}$ and that the likely mechanism involves modulation of Src and phosphatidylinositol 3-kinase signaling pathway. ${ }^{8}$ Constitutive, as opposed to inducible, expression of E-selectin in human dermal microvessels has also been reported ${ }^{9}$ and is linked to T-cell surveillance of vascular surface. ${ }^{10}$ In addition, another potentially important application of E-selectin-targeted imaging is in the detection of early changes in endothelial phenotype linked to atherosclerosis, although prognostic value of E-selectin is controversial. ${ }^{11}$

We previously developed a method of noninvasive imaging of E-selectin expression in endothelial cells. We devised a reporter of E-selectin expression based on nanosized iron oxide particles stabilized by cross-linked dextran (CLIO, crosslinked iron oxide ${ }^{12}$ ). To target E-selectin, we utilized a highly specific, high affinity anti-human E-selectin antibody fragment, $\mathrm{H} 18 / 7 \mathrm{~F}\left(\mathrm{ab}^{\prime}\right)_{2}(1)$, which was 
covalently linked to the surface of CLIO nanoparticles. The experiments with the obtained conjugate, CLIO-F $\left(\mathrm{ab}^{\prime}\right)_{2}$, performed in HUVEC culture suggested that the conjugates were specifically bound to HUVEC only if E-selectin expression was induced by IL- $1 \beta$ treatment. Specific binding of particles to HUVEC resulted in a strong MR T2-weighted signal change. The obtained results suggested that magnetic resonance imaging (MRI) in a combination with a highly superparamagnetic-targeted particle agent is suitable for detecting an inducible expression of cell-adhesion molecule. In vitro experiments subsequently demonstrated that MR imaging enabled imaging of E-selectin expression using the same high-affinity antibody when superparamagnetic particles were substituted with alternative imaging probes: paramagnetic pegylated polymers, ${ }^{13}$ secondary antibody-peroxidase conjugates and polymerizing paramagnetic substrate ${ }^{14}$ or gadoliniumcontaining liposomes. ${ }^{15}$ Subsequently, a different approach that combined a paramagnetic chelated Gd (Gd-DTPA) conjugated with a mannose derivative (mannopyranosyloxyphenyl) phenylacetic acid, ${ }^{16}$ a glycomimetic analog of sLeX) with T1-weighted MR imaging was used towards the goal of E-selectin imaging. ${ }^{17}$ However, the contrast sensitivity of the above paramagnetic agent has been low. ${ }^{18}$

It has been previously demonstrated that precursor blood vessels ('endotubes') preformed in collagen/fibronectin in the absence or in the presence of supporting ('mural') cells, ${ }^{19,20}$ Matrigel, ${ }^{21}$ as well as Matrigel/polymer scaffolds ${ }^{22}$ survive in vivo and connect with the blood supply of the host animal if implanted in immunodefficient mice suggesting an approach for human endothelial cell targeting in mice.

The major goals of the current study were: (1) to investigate whether HUVEC form functional and stable xenogeneic blood neovessels when injected as a suspension in Matrige $^{21}$ into the flanks of $n u / n u$ mice and (2) whether targeted MR probes with the specificity against human endothelial marker (E-selectin) could detect human HUVEC in these vessels in vivo. The ultimate goal of this research was to provide a model system for testing novel human endothelium-specific diagnostic or therapeutic agents in a mouse model.

\section{Materials and methods}

\section{Synthesis of CLIO Conjugate}

The synthesis of iron oxide nanoparticle conjugated to antibody fragments has been described in detail previously. ${ }^{12}$ Briefly, CLIO nanoparticles bearing 2-pyridyldithiopropionyl residues and anti-human E-selectin fragments $\left(\mathrm{H} 18 / 7 \mathrm{~F}\left(\mathrm{ab}^{\prime}\right)_{2}\right.$, a generous gift of Dr Michael Gimbrone Jr, Brigham and Women's Hospital) were linked together by using $N$-succinimidyl $S$-acetylthioacetate (SATA) as a coupling agent.

\section{Cell Culture}

Human umbilical vein endothelial cells (HUVECs) were obtained from the Department of Pathology, Brigham and Women's Hospital (Dr Bill Luscinskas) and propagated in $2 \%$ FBS, complete endothelial cell growth medium (EGM, Cambrex, Baltimore, MD, USA) until confluent. Matrigel (BD Sciences, Bedford, MA, USA) and all additives were thawed and kept at $4^{\circ} \mathrm{C}$ prior to use. Recombinant human bFGF $(50 \mathrm{ng} / \mathrm{ml}$, here and below-final concentration), recombinant human VEGF ( $50 \mathrm{ng} / \mathrm{ml}$, R\&D Systems (Minneapolis, MN, USA) and heparin (128 U/ml, Sigma, St Louis, MO, USA), HUVECs $\left(5 \times 10^{6}\right)$ in $50-80 \mu \mathrm{l}$ of EGM containing serum and supplements were added to the Matrigel mixture (total volume $0.5-0.8 \mathrm{ml}$ ).

\section{Matrigel Injections in Mice}

All animal experiments below were approved by the Massachusetts General Hospital Institutional Animal Care and Use Committee. Athymic female $n u / n u$ mice (Charles River, Stone Ridge, NY, USA), 20-25 g, were anesthetized by intraperitoneal injection of a mixture of Ketamine $(80 \mathrm{mg} / \mathrm{kg})$ and Xylazine $(12 \mathrm{mg} / \mathrm{kg}$ ) and small ice packs were placed next to the flanks for 1-2 min. Using a tuberculin syringe with a hypodermic needle $(22 \mathrm{G})$, Matrigel with HUVECs or alone $(0.6-0.8 \mathrm{ml})$ was injected subcutaneously into the right and left posterior flanks of mice, respectively.

\section{Neovessel Analysis within Matrigel Implants}

Animals harboring HUVEC-containing and control (contralateral) Matrigel implants $(n=2$ each time point) were killed at $4,7,11,14,17$ days after implantation and the implants were dissected without perfusion ( $n=2$ at each time point). Implants were fixed in $4 \%$ paraformaldehyde in PBS, pH 7.4 and examined using light microscopy (Axiovert 100, Zeiss, Wetzlar, Germany). Cryosections $(8 \mu \mathrm{m})$ were either processed for immunofluorescence microscopy (see below) or post-fixed with $4 \%$ formaldehyde/PBS and subjected to fluorescence microscopy using fluorescein excitation mode to visualize strongly autofluorescent erythrocytes (perfused vessels). The number of perfused vessels per $\mathrm{mm}^{2}$ of section was determined by segmentation (IP Lab Spectrum, Signal Analytics). The above sections were further stained with hematoxylin/eosin and imaged using light microscopy. In some experiments, sections were stained by using mouse monoclonal anti-human CD31 antibody (BD Sciences, Bedford, MA, USA), which was labeled with $N$-hydroxysuccinimide ester of digoxigenin carboxylic acid (Roche Diagnostics, Indianapolis, IN, USA). Anti-digoxigenin $\mathrm{F}\left(\mathrm{ab}^{\prime}\right)_{2}$ alkaline phosphatase conjugate (Roche Diagnostics, Indianapolis, 
IN, USA) followed by NBT/BCIP substrate incubation for $30 \mathrm{~min}$ at RT was used for positive identification of human endothelial cells in Matrigel sections in vivo.

\section{Fluorescence Microscopy}

Human endotubes formed in Matrigel in the presence of HUVEC $\left(2.5 \times 10^{5} \mathrm{cells} / \mathrm{ml}\right.$ gel $)$ in vitro were used as positive controls for anti-CD31 staining. Matrigel was overlaid with EGM medium containing growth supplements (Cambrex, Baltimore, MD, USA). Thin sections of endotubes pre-formed in vitro or that of Matrigel implants dissected from mice were post-fixed with $4 \%$ paraformaldehyde (for $5 \mathrm{~min}$ ), blocked with rabbit serum (Vector Laboratories, Burlingame, CA, USA), preincubated with digoxigenin-labeled anti-CD31 antibodies followed by anti-digoxigenin $\mathrm{F}\left(\mathrm{ab}^{\prime}\right)_{2}$ fragment (Roche Applied Science, Indianapolis, IN, USA) custom labeled with Cy3- $N$-hydroxusuccinimide ester (GE Healthcare BioSciences, Piscataway, NJ, USA). Three to four non-consecutive sections were analyzed per each Matrigel sample. Fluorescence images were acquired using appropriate filter sets (Omega Optical, Brattleboro, VT, USA) and a CCD camera (CoolSNAP HQ, Roper Scientific).

\section{Administration of Iron Nanoparticles Conjugated to E-Selectin Antibody Fragment into Mice}

Twenty nmol of human recombinant interleukin (IL-1 $\beta$, Calbiochem were freshly prepared in HBSS, $10 \%$ FBS and $25 \mathrm{mM}$ Hepes, $\mathrm{pH}$ 7.2, and injected IP in a volume of $200 \mu \mathrm{l}$. After $4-6 \mathrm{~h}$, CLIO-F $\left(\mathrm{ab}^{\prime}\right)_{2}$ was injected i.v. through the lateral tail vein at a dose of $3 \mathrm{mg}$ of nanoparticle iron $/ \mathrm{kg}$, using a custom-made $301 / 2$ Gauge catheter. In control experiments, mice $(n=3)$ were injected with iron oxide without pre-injecting with IL- $1 \beta$, stimulated with IL- $1 \beta$ but injected with control conjugate (non-specific $\mathrm{F}\left(\mathrm{ab}^{\prime}\right)_{2}$ ) at the dose of $3 \mathrm{mg}$ iron $/ \mathrm{kg}$.

\section{In Vivo Imaging}

\section{Laser Doppler study}

Laser Doppler imaging was performed in anesthetized animals by using MLDI 5063 (Moore Instruments, Devon, UK) equipped with a $635 \mathrm{~nm}$ laser source. Images of mice at the FOV $=6 \times 6 \mathrm{~cm}$ with the resolution of $128 \times 128$ pixels at $0.5 \mathrm{~mm} /$ pixel were obtained at $37^{\circ} \mathrm{C}$ in a custom-designed airconditioned chamber.

\section{MR imaging}

All MR imaging studies were performed in anesthetized animals using a 7T wide bore Siemens MR system (MGH/MIT/HMS Athinoula A. Martinos Center for Biomedical Imaging). The study included axial, sagittal and coronal T1-weighted (TR/TE $=$ $700 / 20 \mathrm{~ms}$, field of view $6 \mathrm{~cm} \times 6 \mathrm{~cm}$, matrix $=$ $512 \times 512$, number of signal averaging, $\mathrm{NSA}=8$ ) and T2-weighted $(\mathrm{TR}=5000 \mathrm{~ms}$, various $\mathrm{TE}=$ 20-60 ms, FOV $=6 \mathrm{~cm} \times 6 \mathrm{~cm}, \quad$ matrix $=512 \times 512$, NSA =4) spin echo sequence acquisition with a $90^{\circ}$ flip angle followed by $\mathrm{T} 2 *$-weighted gradient echo sequence $(\mathrm{TR}=44 \mathrm{~ms}$, various $\mathrm{TE}=16-100 \mathrm{~ms}$, field of view $=6 \mathrm{~cm} \times 6 \mathrm{~cm}, \quad$ matrix $=256 \times 256$, NSA $=12$ ) using a combined transmit-only surface coil (10 cm diameter loop) and a receive only surface coil ( $5 \mathrm{~cm}$ diameter loop). Animals were imaged before and after the injection of iron oxide nanoparticles (CLIO-F $\left(\mathrm{ab}^{\prime}\right)_{2}$. The negative changes of signal intensity in each Matrigel implant were calculated as:

$$
\Delta \mathrm{SI}=100 \times\left(\mathrm{SI}_{\text {pre }}-\mathrm{SI}_{\text {post }}\right) / \mathrm{SI}_{\text {pre }}
$$

where $\mathrm{SI}_{\text {pre }}$ and $\mathrm{SI}_{\text {post }}$ are regions of interest (ROI) of MR signal intensity before and $3 \mathrm{~h}$ after the injection of CLIO- $\left(\mathrm{Fab}^{\prime}\right)_{2}$, respectively, drawn around Matrigel implants in each flank of the animals. The MR imaging study was performed using in animals either after the IL- $1 \beta$ treatment $(n=10)$ or without the treatment (control, $n=5$ ). A separate control experiment included CLIO linked to the nonspecific, isotype-matched $\mathrm{F}\left(\mathrm{ab}^{\prime}\right)_{2}$ fragment $(n=5)$.

\section{Blood Half-Life and Biodistribution}

To determine blood half-life, radioiodinated ${ }^{125} \mathrm{I}-$ CLIO-F $\left(\mathrm{ab}^{\prime}\right)_{2}$ was used. Two $\mu$ l blood samples were obtained from tail vein at every $15 \mathrm{~min}$ after i.p. injection of IL- $1 \beta$ that was followed by i.v. injection of ${ }^{125}$ I-CLIO-F $\left(\mathrm{ab}^{\prime}\right)_{2}(n=3)$. To evaluate biodistribution of CLIO-F $\left(\mathrm{ab}^{\prime}\right)_{2},{ }^{125} \mathrm{I}$-CLIO-F $\left(\mathrm{ab}^{\prime}\right)_{2}$ was injected to the mice with or without IL- $1 \beta$ pre-treatment. At $2 \mathrm{~h}$ after injection, the Matrigel implants were harvested after perfusing with $4 \%$ formaldehyde in PBS pH 7.4 $(n=4)$. Radioactivity was determined by using a $\gamma$-counter (Compugamma, LKB, Gaithersburg, MD, USA).

\section{Detection of Iron Oxide Nanoparticles in HUVECs by Transmission Electron Microscopy}

Matrigel implants were harvested from mice perfused with PBS pH 7.4, treated with $0.2 \%$ collagenase solution (Worthington, Lakewood, NJ, USA). The labeled cells were isolated by using a magnetic separation column (Macs, Miltenyi Biotech, CA, USA). Cells were with $2 \%$ glutaraldehyde in $0.1 \mathrm{M}$ cacodylate buffer for $1 \mathrm{~h}$ and postfixed in $1 \%$ osmium tetroxide, dehydrated in an alcohol series, and embedded in L.R. White (Electron Microscopy Sciences). Ultrathin sections were examined using Tecnai F20 FEG transmission electron microscope (FEI, Eindhoven, Netherlands). 


\section{Statistical Analysis}

Statistical significance of differences in groups was analyzed by using Student's unpaired, two-tailed test with Welch's correction.

\section{Results}

\section{Anti-E-Selectin Targeted Conjugates}

E-selectin specific MRI probes were synthesized by conjugating anti-human E-selectin $\mathrm{F}\left(\mathrm{ab}^{\prime}\right)_{2}$ antibody fragment $\mathrm{H} 18 / 7^{1}$ to cross-linked iron oxide (CLIO) nanoparticles by using SATA as a coupling agent. Each iron oxide nanoparticle of the resultant conjugate included five molecules of $\mathrm{F}\left(\mathrm{ab}^{\prime}\right)_{2}$, on the average (Figure 1a). The ratio of $\mathrm{F}\left(\mathrm{ab}^{\prime}\right)_{2} / \mathrm{Fe}$ was calculated by determining iron and protein concen-


Figure 1 (A) A schematic drawing showing a cross-linked iron oxide nanoparticle (CLIO) with several $\mathrm{F}\left(\mathrm{ab}^{\prime}\right)_{2}$ molecules tethered to the surface via S-S bonds. (B) Results of atomic force microscopy (AFM) of samples adsorbed on the mica surface (a) CLIO particles modified with 2-pyridyldithiopropionyl groups (average diameter-30 nm) and (b) CLIO conjugated to H18/7 $\mathrm{F}\left(\mathrm{ab}^{\prime}\right)_{2}$ (average diameter-50 $\mathrm{nm}$ ). trations and by assuming that each CLIO iron oxide nanocrystal contained 2048 atoms of iron on the average $^{23}$ and that cross-linking of particles was minimal. The above ratio of $\mathrm{F}\left(\mathrm{ab}^{\prime}\right)_{2} /$ nanoparticle was reached by adding high initial amount of thioacetyl$\mathrm{F}\left(\mathrm{ab}^{\prime}\right)_{2}$ antibody fragment $(1.2 \mathrm{mg}, 11 \mathrm{nmoles})$ per milligram of SPDP- activated CLIO (0.4 mg of iron). This conjugation reaction ratio was higher than that reported previously. ${ }^{12}$ The resultant conjugates had a hydrodynamic diameter of $45 \pm 2.5 \mathrm{~nm}$. The results of laser light scattering (LLS, Table 1) and atomic force microscopy (Figure 1b) showed that the initial increase in size of conjugates did not result in instability of the resultant conjugate. A 1.7-fold increase of hydrodynamic diameter was not followed by appreciably higher transverse relaxivity of iron oxide conjugate (ie R2 values which reflect the ability of these conjugates to shorten $\mathrm{T} 2$ relaxation times of water) if compared to initial particles. However, the binding and internalization of conjugates by IL-1 $\beta$-activated HUVEC showed a threefold higher R2 (142 vs $48(\mathrm{mMs})^{-1}$ ). Hydrodynamic diameter of CLIO-F $\left(\mathrm{ab}^{\prime}\right)_{2}$ conjugates did not change during the storage at $4^{\circ} \mathrm{C}$ for 30 days.

\section{Neovessel Formation within Matrigel Implants In Vivo}

Under conventional ex vivo light microscopy of thick Matrigel sections (Figure 2a, b), neovessels that formed within the Matrigel at 2 weeks after the implantation of cells were clearly visible due to established blood circulation (Figure 2b). Circulation was detectable due to visible light absorption of erythrocytes in microvessels. The formation of these large and branched vessels was observed only in Matrigel implants containing HUVECs, VEGF, FGF-2 and heparin. Neovessels formed in Matrigel alone were scarce, did not show high-order branching (Figure 2a) and appeared to have narrower lumens. The differential vascularization of implants was apparent between 7 and 21 days post implantation. Using strong autofluorescence of erythrocytes as a marker of perfusion (see Figure 2c) we determined that average vascular density in HUVEC Matrigel was higher than in control implants at 2 weeks postimplantation.

Table 1 Physical and biological properties of CLIO- $\left(\mathrm{Fab}^{\prime}\right)_{2}$

\begin{tabular}{|c|c|c|c|}
\hline & Diameter $(\mathrm{nm})^{\mathrm{a}}$ & $\begin{array}{l}R 2\left(m M^{-1} s^{-1}\right), \\
\quad 0.47 T, 40^{\circ} C^{\prime}\end{array}$ & $\begin{array}{l}\text { Blood } T_{1 / 2} \\
\quad(\min )\end{array}$ \\
\hline CLIO-NH ${ }_{2}$ & $27.7 \pm 6.8$ & $55^{\mathrm{b}}$ & $655^{\mathrm{b}}$ \\
\hline CLIO- $\left(\mathrm{Fab}^{\prime}\right)_{2}{ }^{\mathrm{c}}$ & $45.2 \pm 2.5$ & $48 \pm 5$ & $64.0 \pm 0.1^{c}$ \\
\hline CLIO- $\left(\mathrm{Fab}^{\prime}\right)_{2}{ }^{\mathrm{d}}$ & $40.6 \pm 0.2$ & $142 \pm 14^{\mathrm{d}}$ & 二 \\
\hline
\end{tabular}

\footnotetext{
${ }^{a}$ Laser light scattering data, mean \pm s.d.

${ }^{\mathrm{b}}$ Averages, reported in Wunderbaldinger et al (2002). ${ }^{26}$

${ }^{\mathrm{C}}$ This study (in vivo experiment) obtained by using MRI and radioidinated CLIO- $\left(\mathrm{Fab}^{\prime}\right)_{2}$.

${ }^{\mathrm{d}}$ Cell binding in vitro experiment. ${ }^{12}$
} 

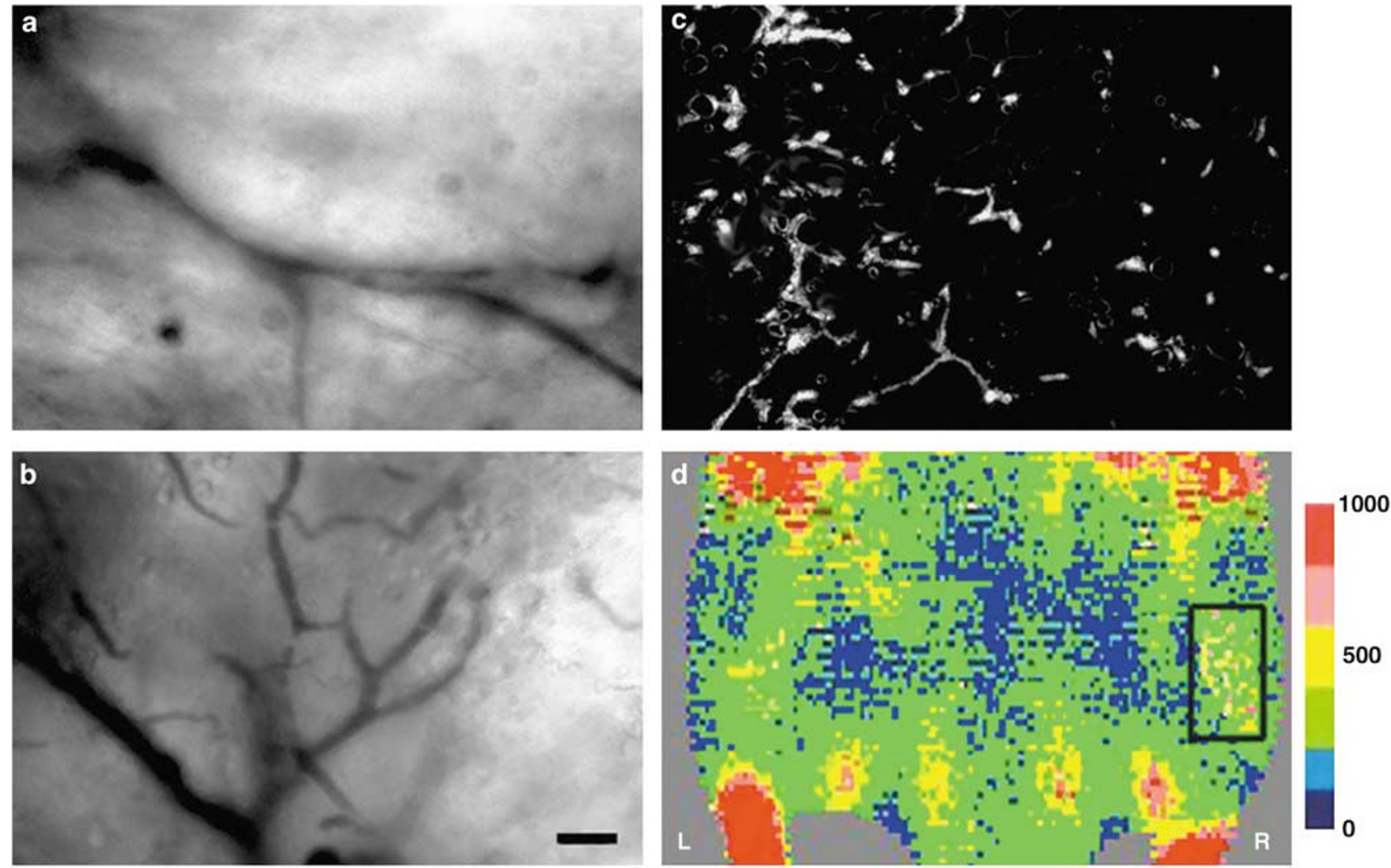

Figure 2 (a) Light microscopy image of a thick section of control Matrigel implant; (b) A section of implant containing HUVEC. (c) Autofluorescence of erythrocytes in HUVEC-seeded implant visualized using FITC filter set. Bar $=250 \mu$ m; (d) color coded laser Doppler flow image of the anterior aspect of a mouse with a HUVEC-containing Matrigel implant in the right flank (boxed) and a control implant in the left flank. Flow rates are color coded (standard perfusion units).

In vivo laser Doppler imaging in mice that harbored Matrigel implants for 14 days demonstrated that if compared to the contralateral side, there was a strong local increase of local blood flow in HUVEC-containing implants (Figure 2d). The increase could be visualized as several high-flow continuous structures (Figure 2d, box, right side of the mouse).

Microscopic analysis of neovessel formation in frozen sections initially included optimization of immunofluorescent detection of human endothelial cells after endotube formation in Matrigel. Thin frozen sections incubated with anti-human CD31 mAb-digoxigenin followed by fluorescent, Cy3labeled anti-digoxigenin antibody showed highly specific fluorescence associated with the surface of the intact endotubes (not shown), as well as small and discrete $(0.2-0.5 \mu \mathrm{m})$ intracellular clusters of CD31 (Figure 3a, 3a inset, arrow). Anti-CD31 staining of endothelial cells in Matrigel at 7 days after the implantation revealed many migrating cells, some of them organized into bundles (Figure $3 \mathrm{~b}$, arrow). At later time points, we observed the formation of vessel-like structures having relatively wide diameters $(25-80 \mu \mathrm{m})$ containing many cells positive for human CD31 assembled in clusters (Figure 3c, inset, arrow). These clusters appeared similar to those detected in endotubes formed in Matrigel in vitro (Figure 3a). Furthermore, the above widediameter HUVEC-positive neovessels showed the presence of red blood cells in their lumens that suggested active circulation of blood in Matrigel implants (Figure 3d).

\section{MR Imaging of Matrigel Implants after the Administration of CLIO-F $\left(\mathbf{a b}^{\prime}\right)_{2}$}

At 2 weeks after the implantation, imaging of neovessels in Matrigel was performed by using T1(Figure 4a,b) and T2-weighted (Figure 4c,d) MRI pulse sequences using a 7T wide-bore imaging system. Initially, imaging in control animals that did not receive iron oxide injections suggested that the sensitivity of the technique was insufficient to detect the presence of neovessels in the implants. Before the injection of superparamagnetic nanoparticles HUVEC-containing (Figure 4a,b arrow) and contralateral implants (Figure 4a, b arrowhead) were nearly isointensive: T1-weighted signal intensity in Matrigel implants seeded with HUVECs was only marginally higher than that of control Matrigel implants. In the absence of iron oxides, T2-weighted images of both implants appeared bright due to the 

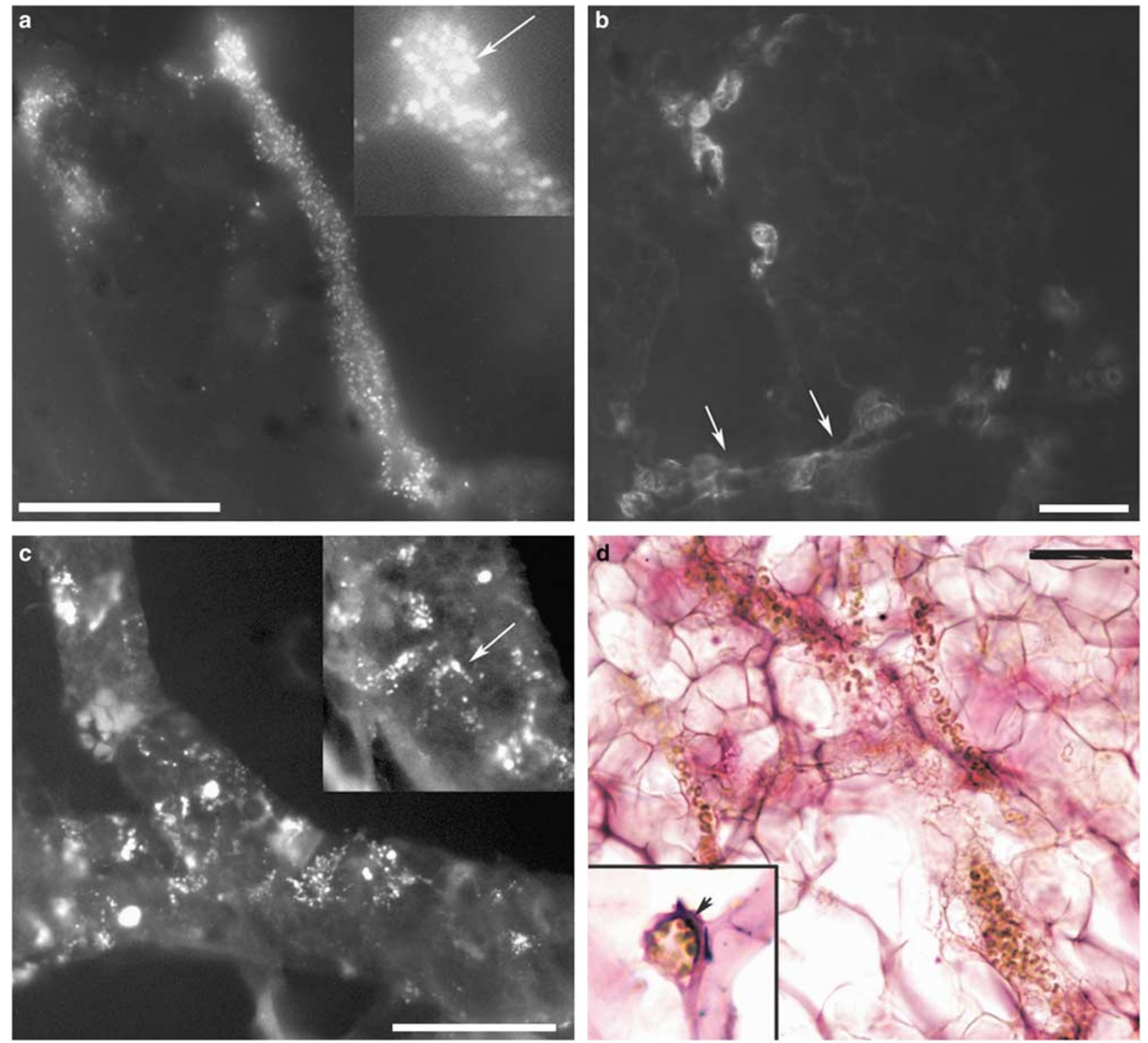

Figure 3 Identification of HUVEC-harboring blood vessels formed in Matrigel. (a) Immunofluorescent image of endotubes formed by HUVEC in Matrigel in vitro. Anti-human CD31 staining was performed in frozen sections as described in Materials and methods. The inset shows clusters of CD31 under a higher magnification $(\times 100)$. (b) Immunofluorescent image (anti-CD31 staining) of human endothelial cells 7 days after the implantation. (c) Same as B, 21 days after implantation. The inset shows clusters stained positively for CD31 resembling those shown in (a). (d) Hematoxylin/eosin staining of section adjacent to one shown in (c). The inset shows a cross section of a blood vessel in Matrigel stained with anti-human CD31 antibody followed by alkaline phosphatase conjugate. Arrow indicates a positive cell in the vascular wall. Bars $=100 \mu \mathrm{m}$.

high water content (Figure $4 d$ ). In contrast, after the consecutive injections of IL- $1 \beta$ (20 nmol/animal) and E-selectin targeted probe CLIO-F $\left(\mathrm{ab}^{\prime}\right)_{2}$ (3 mg $\mathrm{Fe} / \mathrm{kg}$ ) there were significant differences detectable on T2-weighted images (Figure 4c), which showed darkening of HUVEC-seeded implants and no similar change of signal in contralateral implants. By using gradient-echo pulse MRI sequences, we observed highly heterogeneous pattern of the specific signal darkening throughout the volume of the implant (Figure 5c, compare to the control, Figure 5a). The heterogeneity of imaging signal intensity manifested in the presence of extended structures with characteristic dark signal (showed by an arrow, Figure 5b). The signal intensity in animals injected with control, non-targeted, isotype-matched CLIO$\mathrm{F}\left(\mathrm{ab}^{\prime}\right)_{2}$ conjugates did not show any detectable signal intensity changes (Figure 5e-g) in HUVECcontaining or control Matrigel implants. Both control (HUVEC-free) and HUVEC-seeded Matrigel implants were not re-absorbed completely during the course of the experiment and could be easily dissected out after the euthanasia. HUVEC-containing implants dissected from animals that received 



Figure 4 MRI of mice at 7T: $(\mathbf{a}, \mathbf{b})$ a typical T1weighted image obtained before the injection of CLIO particles. Contralateral implants were isointensive on T1-weighted images. (c) T2-weighted image of the mouse treated with i.p. injection of IL-1 $\beta$ followed by E-selectin specific CLIO-F $\left(\mathrm{ab}^{\prime}\right)_{2}$, (d) T2-weighted image of a control mouse that received no IL-1 $\beta$ injection. Images were obtained before and after $3 \mathrm{~h}$ of CLIO-F $\left(\mathrm{ab}^{\prime}\right)_{2}$ nanoparticles injection as described in Materials and methods. The location of HUVEC-containing implants is shown with arrows; contralateral (control) implants are shown with arrowheads. (a, c, d) Coronal oblique views; (b) axial view of the animal.
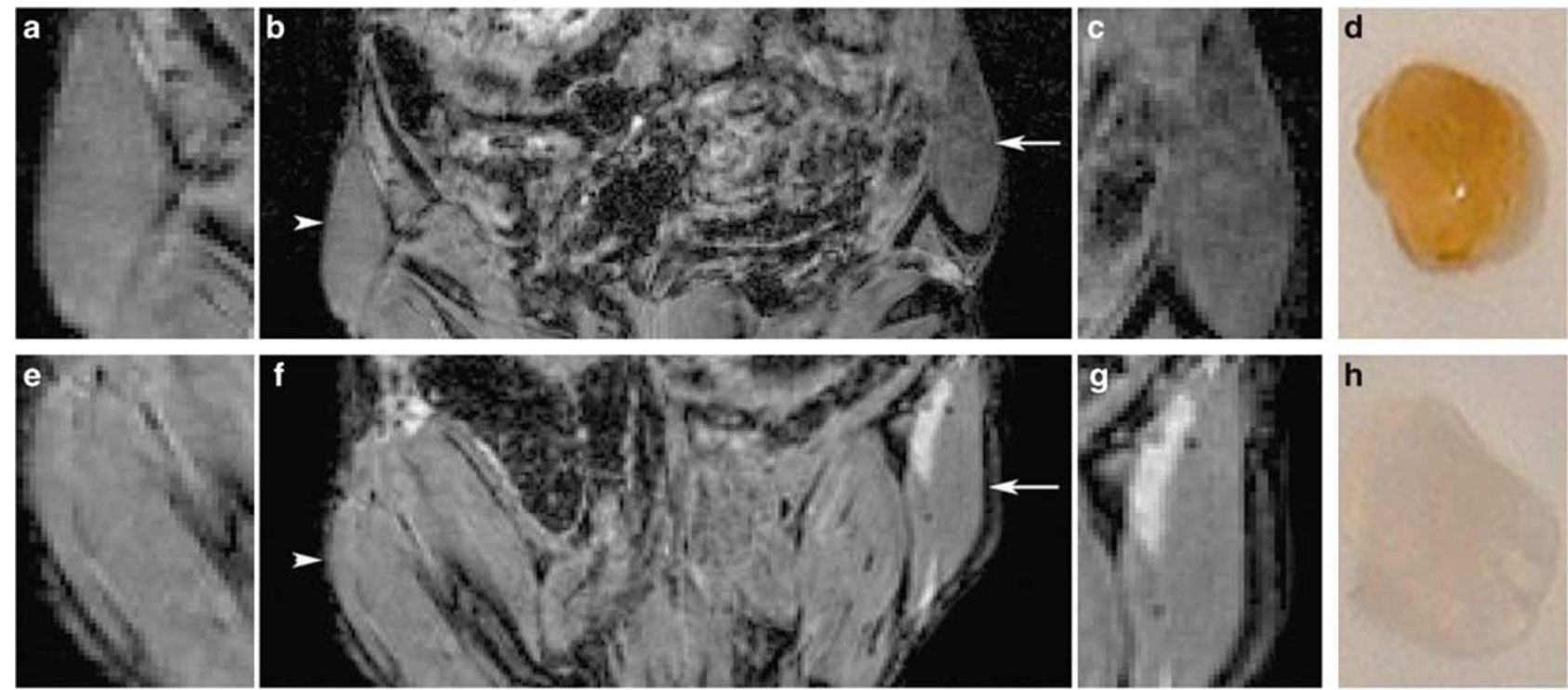

Figure $5 \mathrm{~T}^{*}$-weighted gradient-echo (GRE) MR images of mice bearing Matrigel implants (a-c and e-g). Implants containing Matrigel alone are shown on the left (a, enlarged, shown on $\mathbf{b}, \mathbf{f}$ by arrowheads), HUVEC-containing implants are shown on the right (e, enlarged, shown on $\mathbf{b}, \mathbf{f}$ by arrows). Images were obtained either $3 \mathrm{~h}$ after the injection of anti-E-selectin CLIO-F(ab') $)_{2}$ and (images $\left.\mathbf{a}-\mathbf{c}\right)$ or of nonspecific CLIO-F $\left(\mathrm{ab}^{\prime}\right)_{2}$ nanoparticles (images $\left.\mathbf{e}-\mathbf{g}\right)(\mathrm{bars}=100 \mu \mathrm{m})$. Shown also are light photography images of HUVEC seeded implant $(\mathbf{d})$ and a control implant (h), surgically removed from euthanized mouse injected with E-selectin specific CLIO-F $\left(\mathrm{ab}^{\prime}\right)_{2}$.

both IL-1 $\beta$ and E-selectin specific CLIO-F $\left(\mathrm{ab}^{\prime}\right)_{2}$ nanoparticles were strikingly different in appearance from control implants (see Figure $5 \mathrm{~d}$ and $\mathrm{h}$ ). Unlike the controls, they were non-transparent and showed dark coloration. The quantitation of MR signal intensity enabled to establish the percentage change of signal intensity in the Matrigel implants before the injection and $3 \mathrm{~h}$ that followed the 
606
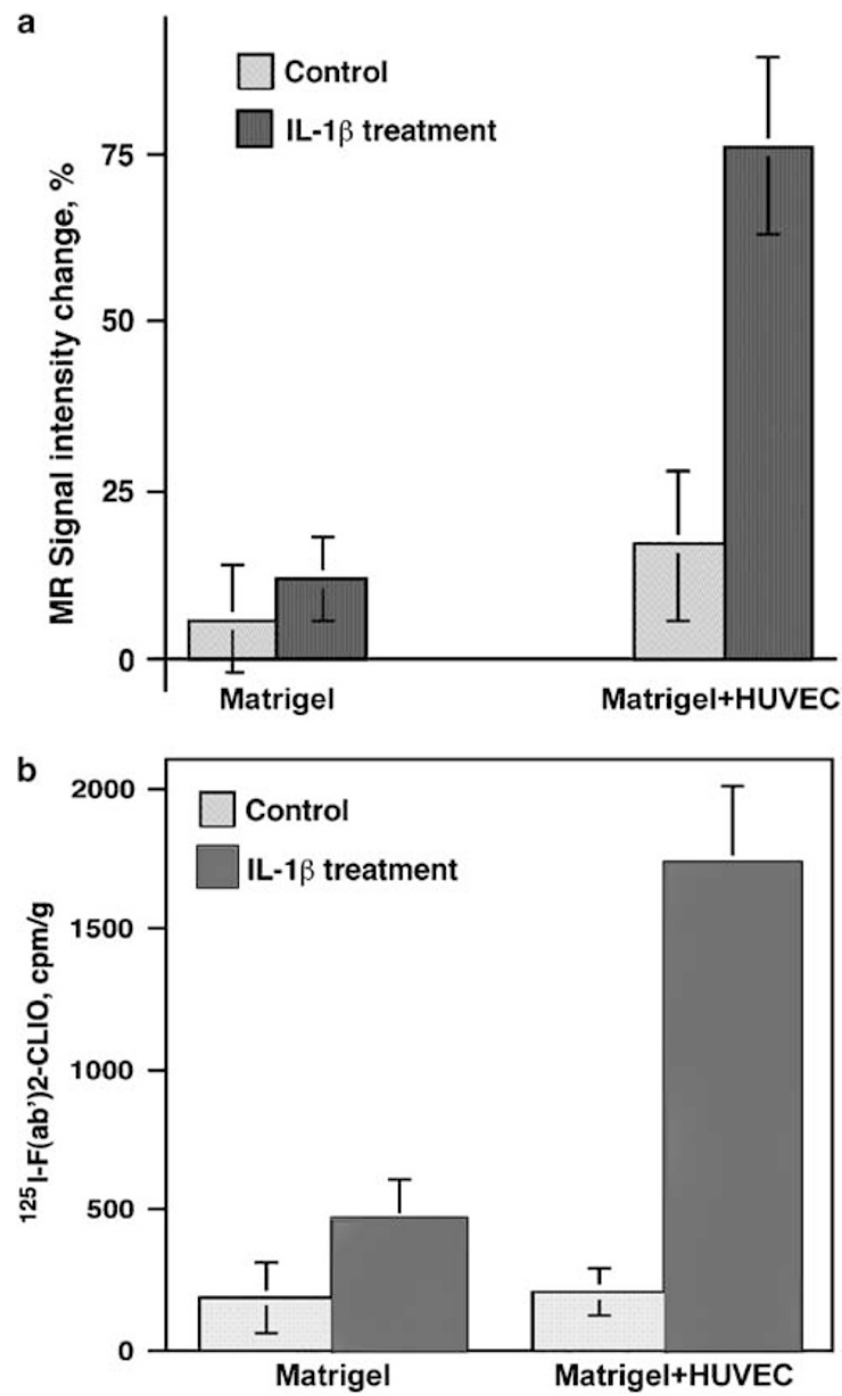

Figure 6 (a) Region-of-interest measurements of MR signal intensity decrease before and after the injection of CLIO-F $\left(\mathrm{ab}^{\prime}\right)_{2}$ nanoparticles measured in HUVEC-containing or control implants of animals that were either treated with IL-1 $\beta$ (solid bars, $n=10$ ) or in control animals (shaded bars, $n=5$ ). Data are shown as mean \pm s.e.m. The differences in HUVEC-containing Matrigel implanted animals were statistically significant $(P<0.01)$. The differences in Matrigel-only animals were insignificant $(P>0.05)$. (b) Biodistribution data in animals that received i.v. injections of ${ }^{125}$ I-labeled CLIO-F $\left(\mathrm{ab}^{\prime}\right)_{2}$ either after the IL- $1 \beta$ treatment $(n=5$, solid bars) or without treatment $(n=5$, shaded bars). Radioactivity associated with Matrigel implants was quantitatively analyzed by using $\gamma$-counting.

injection of CLIO-(Fab') $)_{2}$ (Figure 6a). Negative signal intensity measured in animals treated with IL-1 $\beta$ was higher than in control animals in both HUVECand control Matrigel implants. The implants containing HUVECs treated with IL- $1 \beta$ showed six times greater imaging signal change after the injection of E-selectin targeted CLIO-F $\left(\mathrm{ab}^{\prime}\right)_{2}$ if compared to signal intensities in the contralateral control implants in the same animals. In control animals that did not receive IL- $1 \beta$, signal change due to nonspecific delivery and uptake of nanoparticles was insignificant. The MR signal measurements (Figure 6a) were supported by a biodistribution study (Figure 6b): (1) there was, on the average, more ${ }^{125}$ I-labeled CLIO-F $\left(\mathrm{ab}^{\prime}\right)_{2}$ particles that accumulated in both control and experimental implants after the IL-1 $\beta$ treatment than in the absence of IL-1 $\beta$ treatment; (2) radioiodinated CLIO-F $\left(\mathrm{ab}^{\prime}\right)_{2}$ showed an approximately four-fold higher binding in the HUVEC-positive implants than in the control ones in the case of IL- $1 \beta$ treated animals. There was no difference in targeted iron oxide binding to implants in non-treated mice (Figure 6b).

\section{Histology and Microscopy}

After staining of Matrigel histology sections with Prussian blue and hematoxylin, vessels that were highly positively stained for iron were detected only in Matrigel implants initially seeded with HUVECs (Figure 7b, arrows, compare with Figure 7a). The majority of detectable (stained) iron oxide was localized inside the lumena of neovessels.

Further study of E-selectin targeted CLIOs accumulation in HUVECs included transmission electron microscopy (TEM) of ultrathin sections (Figure 7c). The analysis of TEM images showed strongly electron-dense particle clusters $40-80 \mathrm{~nm}$ in diameter. Only internalized CLIO-F $\left(\mathrm{ab}^{\prime}\right)_{2}$ particles were visible on micrographs due to the fact that cell surface-bound particles were removed during cell isolation for microscopy. The electron-dense clusters were not present in the majority of endothelial cells harvested from control animals that did not receive injections of nanoparticles. The uptake of CLIO particles in cells constituted $25 \%$ of total cellassociated number of particles.

\section{Discussion}

The refinement of human endothelial cell adoptive transfer techniques has been recently attracting attention of vascular and tumor biologists. ${ }^{19-22,24}$ However, the recent high level of interest to the above topic is not limited exclusively to the goals of vascular engineering, for example, at establishing functional blood supply from the host to the vascular graft or implant. Stable neovessels bearing differentiation markers of functional human endothelium linked to the blood supply of the host could serve as excellent model targets for new therapies and imaging probes that need to be tested in live animals.

Previous research reported by Schechner et a ${ }^{19}$ demonstrated that human endotubes pre-formed in simple mixed collagen I/human plasma fibronectin gels could be implanted into SCID mice resulting in functional blood supply after the maturation of the endotubes and formation of neovessels. The overexpression of anti-apoptotic Bcl-2 in $\mathrm{HUVEC}^{24}$ or co-implantation of HUVEC alongside with mesen- 

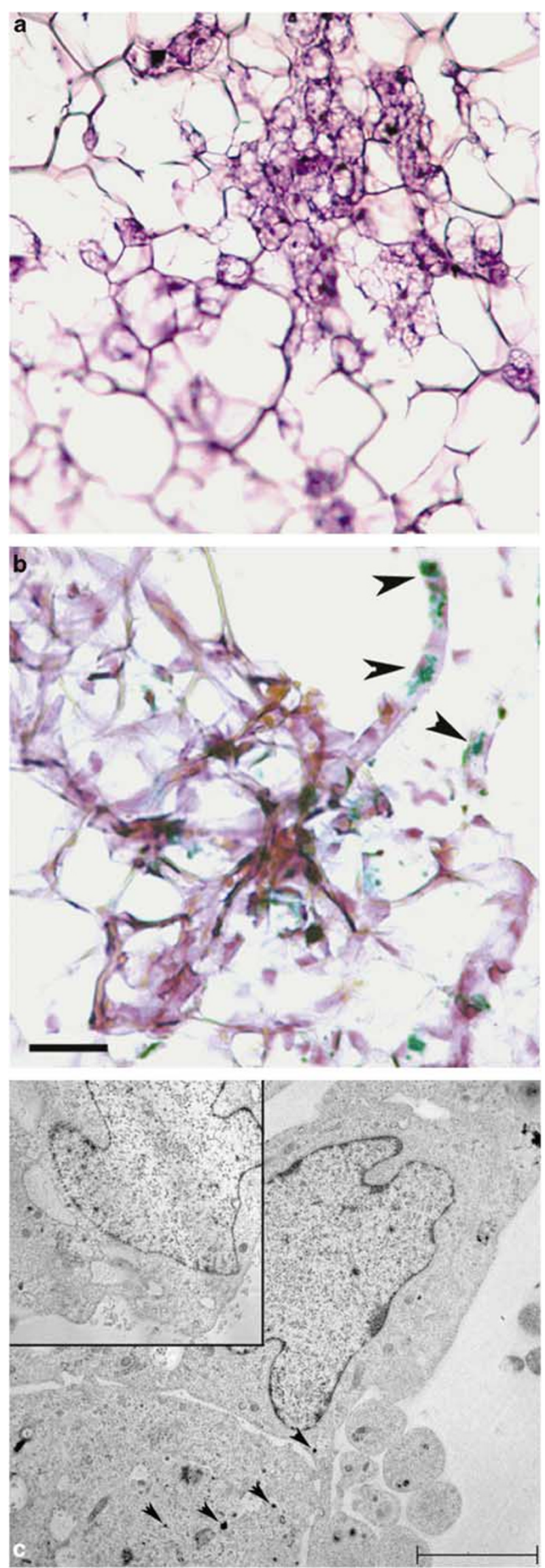

chymal precursor cells ${ }^{20}$ resulted in a stabilization of above HUVEC neovascular xenotransplants. Furthermore, an injection of HUVEC suspended in Matrigel in Rag2-/- mice also resulted in a stable expression of differentiation markers by human endothelial cells and showed an evidence of circulation. ${ }^{21}$ In both mouse models reported so far engineered human vessels survived up to 80 days in mice and showed the presence host mesenchymal cells that support human endothelium. In current study, we chose to use Matrigel as a temporary scaffold to guide new vessel formation, growth and organization in the presence of VEGF and FGF-2. This allowed suspending cell in a fluid matrix, which can be then subcutaneously injected. The gelation rate of resultant implants could be easily controlled by local hypothermia. The implanted HUVECs had aligned and formed large diameter endotubes and neovessels that showed high order branching, in contrast to those formed after the plain Matrigel injections (Figure 2). Initially, histology showed an evidence of CD-31 positive human endothelial cells that formed structures resembling endotubes occurring in Matrigel in vitro (Figure $3 a, b)$. These thin structures matured into widediameter branched structures (Figure 3c). Histology evidence of functional blood supply (Figure 3d) suggested that functional capillaries were forming in the implants and that either human endothelial cells were still present and functional, or that human endothelium served as a guide for mouse endothelial cells that eventually formed mixed (mosaic) neovessels containing both human and mouse cells.

To test whether: (1) the neovessels still contained functional human cells that were showing inducible expression of E-selectin post implantation, (2) the presence of these cells could be identified in vivo with imaging, we used previously developed antihuman E-selectin antibody fragment conjugated with iron oxide nanoparticles (Figure 1). The imaging at high field (7T vs clinical magnet strength of 1.5T) enabled to achieve higher signal-to-noise ratios at high field homogeneity and without significant image distortion. MRI in vivo demonstrated that E-selectin-targeted nanoparticles were consistently showing specific 'darkening' of Matrigel implants that contained HUVEC, in contrast to the contralateral control implants (Figure 5). How-

Figure $7 E_{X}$ vivo analysis of Matrigel implants $(20 \mathrm{~d}$ after implantation) isolated from mice $3 \mathrm{~h}$ after i.v. administration of CLIO-F $\left(\mathrm{ab}^{\prime}\right)_{2}$ conjugates at the dose equivalent to $3 \mathrm{mg} / \mathrm{kg}$ of $\mathrm{Fe}$ that followed i.p. treatment with IL-1 $\beta$. The tissues were perfused with $4 \%$ formaldehyde in PBS, frozen sectioned, stained with Perl' $^{\prime}$ reagent and hematoxylin/eosin. (a) Control (HUVEC-free) Matrigel implants; (b) HUVEC-containing implants. Prussian blue stain was detected only in HUVEC-containing implants. Bar $=100 \mu \mathrm{m}$. (c) Transmission electron microscopy of internalized iron oxide nanoparticles in HUVECs that were isolated from Matrigel implants. Inset-control HUVEC cell. Electron-dense clusters are indicated by arrowheads. Magnification $\times 15000$. Bar $=1 \mu \mathrm{m}$ 
ever, we assumed that IL-1 $\beta$ treatment could potentially result in additional cytokine-mediated activation of non-specific adsorptive endocytosis or pinocytosis of particles by endothelial cells that were maintained in Matrigel scaffolds. Alternatively, the local increase of vascular permeability could potentially result in non-specific leakage and retention of particles in the implants. Thus, we tested this possibility in experiments that used control nanoparticle probes, which were linked to non-specific isotype-matched antibody fragment. In the latter case, no darkening of HUVEC-seeded Matrigel was visible even after the animals were treated with IL-1 $\beta$ (Figure 5e-g). The observed difference in signal on MR images was quantified by using region-of-interest analysis and compared to biodistribution data that suggested approximately a three times higher accumulation and retention of iron oxide nanoparticles in HUVEC-containing Matrigel implants and a six-fold stronger T2weighted MR signal change (Figure 6). The above effect, that is, a higher MR signal difference measured in the HUVEC-containing and in the control implants than the difference suggested by biodistribution, could be explained by the measurements shown in Table 1 and previous observations made in tranferrin-targeted iron oxide nanoparticle uptake experiments. ${ }^{25}$ Upon cell binding the particles get taken up by the cells with the concomitant increase in iron R2 relaxivity because of the intracellular clustering inside the cells (Table 1). The evidence of such uptake is presented in Figure 7 that shows specific staining for iron in neovessels and in endothelial cells that were incubated with anti-E-selectin targeted iron oxides.

In conclusion, we: (1) showed that human endothelial cells support functional blood vessels in Matrigel implants in athymic mice for at least 21 days; (2) tested the model of adoptive endothelial cell transfer for targeting of IL- $1 \beta$ activated endothelial cells using a novel MR reporter probe directed to human E-selectin. The obtained results suggested that mouse models of adoptive endothelial cell transfer in mice for evaluating novel targeted agents that could be detected by imaging.

\section{Acknowledgements}

The authors acknowledge Dr Michael Gimbrone Jr (Department of Pathology, Brigham and Women's Hospital, Boston, MA) and Jean-Marie Kiely (same Department) for generously providing H18/7 antibody fragment, Kay Case for providing HUVEC (Dr Bill Luscinskas, Vascular Research Division, Department of Pathology, Brigham and Women's Hospital, Boston, MA), Drs Lee Josephson and Nikolay Sergeyev (CMIR, Massachusetts General Hospital) for providing PDP-CLIO. This work has been supported in part by NIH 1P50CA86355-01 (Project 2) and 5RO1 CA74424-01. Denise Torres was supported by NIH Research Supplement for Underrepresented Minorities.

\section{References}

1 Bevilacqua MP, Pober JS, Mendrick DL, et al. Identification of an inducible endothelial-leukocyte adhesion molecule. Proc Natl Acad Sci USA 1987;84: 9238-9242.

2 Bischoff J, Brasel C, Kraling B, et al. E-selectin is upregulated in proliferating endothelial cells in vitro. Microcirculation 1997;4:279-287.

3 Aoki M, Kanamori M, Yudoh K, et al. Effects of vascular endothelial growth factor and E-selectin on angiogenesis in the murine metastatic rct sarcoma. Tumour Biol 2001;22:239-246.

4 Nguyen M, Corless CL, Kraling BM, et al. Vascular expression of E-selectin is increased in estrogenreceptor-negative breast cancer: A role for tumor-cellsecreted interleukin-1 alpha. Am J Pathol 1997;150: 1307-1314.

5 Hebbar M, Peyrat JP. Significance of soluble endothelial molecule e-selectin in patients with breast cancer. Int J Biol Markers 2000;15:15-21.

6 Sheen-Chen SM, Eng HL, Huang CC, et al. Serum levels of soluble E-selectin in women with breast cancer. Br J Surg 2004;91:1578-1581.

7 Koch AE, Halloran MM, Haskell CJ, et al. Angiogenesis mediated by soluble forms of E-selectin and vascular cell adhesion molecule-1. Nature 1995;376:517-519.

8 Kumar P, Amin MA, Harlow LA, et al. Src and phosphatidylinositol 3-kinase mediate soluble E-selectin-induced angiogenesis. Blood 2003;101:3960-3968.

9 Kluger MS, Johnson DR, Pober JS. Mechanism of sustained E-selectin expression in cultured human dermal microvascular endothelial cells. J Immunol 1997;158:887-896.

10 Chong BF, Murphy JE, Kupper TS, et al. E-selectin, thymus- and activation-regulated chemokine/ccl17, and intercellular adhesion molecule-1 are constitutively coexpressed in dermal microvessels: A foundation for a cutaneous immunosurveillance system. J Immunol 2004;172:1575-1581.

11 Roldan V, Marin F, Lip G, et al. Soluble E-selectin in cardiovascular disease and its risk factors. A review of the literature. Thromb Haemost 2003;90:1007-1020.

12 Kang HW, Josephson L, Petrovsky A, et al. Magnetic resonance imaging of inducible E-selectin expression in human endothelial cell culture. Bioconjug Chem 2002;13:122-127.

13 Kang H, Weissleder R, Bogdanov AJ. Targeting of mpeg-protected polyamino acid carrier to human E-selectin in vitro. Amino Acids 2002;23:301-308.

14 Bogdanov Jr A, Matuszewski L, Bremer C, et al. Oligomerization of paramagnetic substrates result in signal amplification and can be used for mr imaging of molecular targets. Mol Imag 2002;1:16-23.

15 Mulder WJ, Strijkers GJ, Griffioen AW, et al. A liposomal system for contrast-enhanced magnetic resonance imaging of molecular targets. Bioconjug Chem 2004;15:799-806.

16 Kogan T, Dupre B, Keller K, et al. Rational design and synthesis of small molecule, non-oligosaccharide selectin inhibitors: ( $\alpha$-D-mannopyranosyeoxy) biphenyl-substituted carboxylic acids. J Med Chem 1995;38: 4976-4984. 
17 Sibson NR, Blamire AM, Bernades-Silva M, et al. Mri detection of early endothelial activation in brain inflammation. Magn Reson Med 2004;51: 248-252.

18 Barber PA, Foniok T, Kirk D, et al. Mr molecular imaging of early endothelial activation in focal ischemia. Ann Neurol 2004;56:116-120.

19 Schechner JS, Nath AK, Zheng L, et al. In vivo formation of complex microvessels lined by human endothelial cells in an immunodeficient mouse. Proc Natl Acad Sci USA 2000;97:9191-9196.

20 Koike N, Fukumura D, Gralla O, et al. Tissue engineering: creation of long-lasting blood vessels. Nature 2004;428:138-139.

21 Skovseth DK, Yamanaka T, Brandtzaeg P, et al. Vascular morphogenesis and differentiation after adoptive transfer of human endothelial cells to immunodeficient mice. Am J Pathol 2002;160:1629-1637.
22 Levenberg S, Golub J, Amit M, et al. Endothelial cells derived from human embryonic stem cells. Proc Natl Acad Sci USA 2002;99:4391-4396.

23 Shen T, Weissleder R, Papisov M, et al. Monocrystalline iron oxide nanocompounds (MION): physicochemical properties. Magn Reson Med 1993;29: 599-604.

24 Enis DR, Shepherd BR, Wang Y, et al. Induction, differentiation, and remodeling of blood vessels after transplantation of Bcl-2-transduced endothelial cells. Proc Natl Acad Sci USA 2005;102:425-430.

25 Moore A, Basilion JP, Chiocca EA, et al. Measuring transferrin receptor gene expression by NMR imaging. Biochim Biophys Acta 1998;1402:239-249.

26 Wunderbaldinger P, Josephson L, Weissleder R. Tat peptide directs enhanced clearance and hepatic permeability of magnetic nanoparticles. Bioconjug Chem 2002;13:264-268. 